\title{
Normal mitochondrial structure and genome maintenance in yeast requires the dynamin-like product of the $M G M 1$ gene
}

\author{
Kunliang Guan $^{1}$, Lynn Farh ${ }^{2}$, Tricia K. Marshall ${ }^{2}$, Robert J. Deschenes ${ }^{2}$ \\ 1 Department of Biochemistry, University of Michigan, Ann Arbor, Michigan 48109-0606, USA \\ 2 Department of Biochemistry, University of Iowa, Iowa City, IA 52242, USA \\ Received: 26 October $1992 /$ Accepted: 20 December 1992
}

\begin{abstract}
The isolation and characterization of $M G M 1$, an yeast gene with homology to members of the dynamin gene family, is described. The MGM1 gene is located on the right arm of chromosome XV between STE4 and $P T P 2$. Sequence analysis revealed a single open reading frame of 902 residues capable of encoding a protein with an approximate molecular mass of $101 \mathrm{kDa}$. Loss of MGM1 resulted in slow growth on rich medium, failure to grow on non-fermentable carbon sources, and loss of mitochondrial DNA. The mitochondria also appeared abnormal when visualized with an antibody to a mitochondrial-matrix marker. $M G M 1$ encodes a dynaminlike protein involved in the propagation of functional mitochondria in yeast.
\end{abstract}

Key words: Saccharomyces cerevisiae - Dynamin - Mitochondria - GTP binding protein

\section{Introduction}

The assembly and propagation of functional mitochondria require coordinate input from the nuclear and mitochondrial genomes. In yeast, a large number of mutants affecting mitochondrial function have been isolated and characterized (Dujon 1981; Tzagoloff and Dieckmann 1990). Since yeast is able to grow anaerobically, cells with severely defective mitochondria are still viable if grown on fermentable carbon sources. For example, mutants that have lost the entire mitochondrial genome $\left(\varrho^{\circ}\right)$ still grow anaerobically. Although the mitochondrial genome is dispensable under these conditions, mitochondrial functions encoded in the nucleus are required. Nuclear and mitochondrial gene mutations that lead to the generation of $\varrho^{\circ}$ mutant strains have been described. The proteins encoded by these genes include those required for mitochondrial DNA replication and mitochondrial protein synthesis (Myers et al. 1987; Tzagoloff and

Correspondence to: R. J. Deschenes
Dieckmann 1990). Recently, Jones and Fangman (1992) isolated a gene from Saccharomyces cerevisiae, MGM1, that is predicted to encode a protein with sequence similarity to members of the dynamin protein family. Loss of $M G M 1$ results in the loss of the mitochondrial genome. In this report, we describe a further characterization of the $M G M 1$ gene and a sequence that differs from the one reported by Jones and Fangman (1992). Immunofluorescence studies using an antibody to a mitochondrial matrix-localized protein (hsp58) revealed that the morphology and intracellular localization of mitochondria is affected by the loss of Mgm1p.

The first dynamin protein to be described, D100, was isolated from bovine brain by virtue of its property of being released from microtubules on the addition of ATP (Shpetner and Vallee 1989; Scaife and Margolis 1990). However, a number of properties distinguish dynamins from true microtubule motor proteins (Vale et al. 1985a, b; Schnapp and Reese 1989; Vallee et al. 1989). Dynamins bind GTP, rather than ATP which is the preferred nucleotide triphosphate for kinesin and dynein microtubule motors. Although microtubules have been shown to stimulate dynamin GTPase activity (Shpetner and Vallee 1992), it has not been possible to observe a microtubuledependent mechanochemical motor activity using highlypurified dynamin preparations.

The isolation and characterization of Drosophila and yeast dynamin mutants has revealed a number of cellular processes mediated by dynamin protein. A yeast dynamin-like gene, VPS1, was identified in a genetic screen for mutants defective in vacuolar protein sorting (Rothman and Stevens 1986; Rothman et al. 1990). In addition to mis-sorting of proteins destined for the vacuole, the vps 1 mutant displays abnormal intracellular membrane organization and the cells are temperature-sensitive for growth. VPS1 was independently isolated in a screen for sporulation mutants and named SPO15 (Yeh et al. 1986). SPO15 mutants initiate the early events of meiosis, such as recombination, but arrest prior to the meiotic division. The protein encoded by VPS1 (SP015) is $45 \%$ identical to the rat brain dynamin, D100 (Obar et al. 1990; Roth- 
man et al. 1990; Yeh et al. 1991) period Mutations in the Drosophila gene shibire result in a reversible temperaturesensitive paralysis. Shibire encodes a dynamin homolog with $69 \%$ amino-acid sequence identity to rat D100 (Chen et al. 1991; Poodry and Edgar 1979; van der Bliek and Meyerowitz 1991). Paralysis results from an endocytic defect at the nerve terminus. Thus, the mutant phenotypes of the dynamin genes characterized to-date suggest roles for dynamins in vesicle trafficking, endocytosis, and sporulation. Our study and that of Jones and Fangman (1992) suggest that a role for dynamin-like proteins in mitochondrial function be added to the list. Deletion of $M G M 1$ causes respiratory deficiency, loss of mitochondrial DNA, and altered mitochondrial morphology.

\section{Materials and methods}

Yeast strain construction. The yeast strains used in this study are listed in Table 1. Standard procedures for yeast mating, sporulation, and scoring of tetrads were used (Sherman et al. 1986). Yeast was grown in either rich medium [YEPD; $1 \%$ yeast extract, $2 \%$ Bactopeptone (Difco Laboratories)] or synthetic medium (SC-AA: $0.67 \%$ yeast nitrogen base without amino acids) supplemented with amino acids to satisfy auxotrophic requirements. Glucose (2\%) was used as the carbon source except in the experiments testing growth on non-fermentable carbon sources in which case glycerol $(2 \%)$ and ethanol $(2 \%)$ were used. Yeasts were transformed by the lithium acetate procedure (Ito et al. 1983).

Cloning and sequence analysis. The 14-kb lambda clone that harbors $M G M 1$, was originally isolated from a $\lambda$-dash yeast genomic library (Stratagene) based on homology to a DNA probe from a protein tyrosine phosphatase gene (PTP2), designated QGP5 (Guan et al. 1992). The QGP5 probe was obtained by the polymerase chain reaction (PCR) using two degenerate oligonucleotides to conserved regions found in tyrosine phosphatases. Details concerning this probe can be found in previous reports (Guan et al. 1991, 1992). The YPTP2-4 clone was mapped by restriction digestion and fragments were subcloned into either M13mp18 or M13mp19 for sequence determination. A series of synthetic oligonucleotides were used as sequence primers to derive a complete sequence of both strands of the gene. Sequencing was performed by the dideoxy chain-termination method using Sequenase (US Biochemicals). Sequence analysis was performed using the Wisconsin GCG software package and Microgenie (Beckman). Unless otherwise noted, all DNA modifying enzymes were purchased from New England Biolabs. Standard procedures were used for DNA manipulations (Sambrook et al. 1989).

Plasmid construction and gene replacement. MGM1 was subcloned into pUC19 to obtain pMGM1. The clone pMGM1 encompasses $4.47 \mathrm{~kb}$ of DNA that includes 121 bases upstream of the proposed initiator ATG, and 1.6-kb downstream from the translation stop. To create an $M G M 1$ gene disruption with the $L E U 2$ gene, pMGM1 was digested with $P_{s t} \mathrm{I}$, and the resulting $3^{\prime}$ overhangs were converted to blunt ends with T4 DNA polymerase. A $2.2-\mathrm{kb}$ XhoI-SalI fragment of $L E U 2$ was cut from YEp13. The ends of the LEU2 fragment were converted to blunt ends with the Klenow fragment of DNA polymerase and ligated to the Pst -cut pMGM1 plasmid (see Fig. 1). To create an $M G M 1$ gene disruption with the $U R A 3$ gene, pMGM1 was cut with HindIII and a 1.2-kb HindIII fragment of yeast $U R A 3$, isolated from YEp24, was inserted. Gene disruptions were performed by transforming the indicated diploid with linear fragments of DNA, using a lithium acetate transformation protocol (Ito et al. 1983; Rothstein 1983). The integration sites were confirmed by Southern blots (Southern 1975) using yeast DNA
Table 1. Strains used in this study

\begin{tabular}{|c|c|}
\hline Strain & Genotype \\
\hline RJY264a & 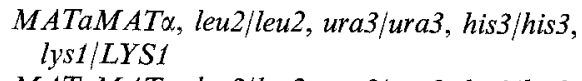 \\
\hline RJY310 a & $\begin{array}{l}\text { MATaMATx, leu2/leu2, ura3/ura3, his } 3 / \text { his3, } \\
\text { lys1/LYS1 }\end{array}$ \\
\hline RJY389a & 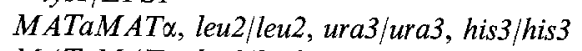 \\
\hline RJY382 ${ }^{\mathrm{b}}$ & $\begin{array}{l}\text { MATaMATo, leu2/leu2, ura3/ura3, his3/his3, } \\
\quad \text { lys1/LYS1, MGM1/mgm1::URA3 }\end{array}$ \\
\hline RJY390 & 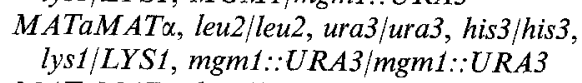 \\
\hline RJY $463^{d}$ & $\begin{array}{l}\text { MATaMATx, leu } 2 / \text { leu } 2, \text { ura3/ura3, his } 3 / \text { his } 3, \\
\text { MGM1/mgm1::LEU2 }\end{array}$ \\
\hline RJY $555^{\mathrm{e}}$ & 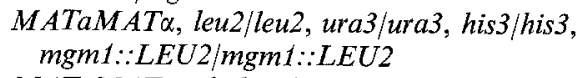 \\
\hline RJY545 & 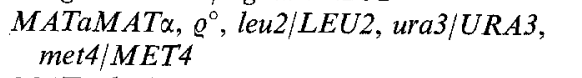 \\
\hline RJY386 & $M A T a$, leu 2, ura3, his 3, mgm1::URA3 \\
\hline RJY387g & MATa, leu2, ura3, his3 \\
\hline RJY559 ${ }^{\mathrm{h}}$ & MATa, leu2, ura3, his 3 \\
\hline RJY $560^{\mathrm{h}}$ & MATa, leu2, ura3, his3 \\
\hline RJY561 ${ }^{\text {h }}$ & $M A T \alpha$, leu2, ura3, his 3, mgm1 $:$ LEU2 \\
\hline RJY562 ${ }^{\mathrm{h}}$ & $M A T \alpha$, leu2, ura3, his3, mgm1::LEU2 \\
\hline KL14-4B ${ }^{i}$ & $M A T \alpha, \varrho^{\circ}$ \\
\hline
\end{tabular}

a Laboratory diploid strains

b Obtained by transformation of RJY264

c Obtained by crossing segregants derived from sporulating RJY382

d Obtained by transformation of RJY389

e Obtained by crossing segregants derived from sporulating RJY463

f KL14-4B was crossed with RJY387, the resulting dipoid was sporulated and two $\varrho^{\circ}$ segregants were crossed to create the homozygous $\varrho^{\circ}$ diploid

g Obtained by sporulating RJY382

h Obtained by sporulating RJY463

i From M. Crivellone; see Myers et al. (1987)

isolated by the method of Hoffman and Winston (1987). The plasmid pMGM1 was labeled by the random primer method using ${ }^{32} \mathrm{P}$-deoxyadenosine triphosphate (Amersham). The filters were exposed using Kodak XAR film with an intensifying screen.

RNA isolation and Northern hybridization. Total yeast RNA was isolated from an exponentially-growing culture of RJY310 following the method of Schmitt et al. (1990). Poly A ${ }^{+}$RNA was purified by oligo $\mathrm{d}(\mathrm{T})$-Sepharose chromatography (Pharmacia LKB Biotech.). The RNA was resolved by electrophoresis in a 1.2\% agaroseformaldehyde gel, transferred to a nitrocellulose membrane, and hybridized with the ${ }^{32} \mathrm{P}$-labeled $M G M 1$ probe derived from labeling the 3-kb BamHI fragment of $M G M 1$. The transcript size was estimated by comparison to RNA molecular weight standards (Bethesda Research Labs) loaded in an adjacent lane.

Protein immunoblot. Total protein extracts were prepared from RJY389 and RJY390 essentially as described (Deschenes and Broach 1987; Stimmel et al. 1990). Total cellular protein was separated by SDS-PAGE on a $12.5 \%$ gel and electrophoretically transferred to Westran (Schleicher and Shuell). The filter was probed with anti-hsp58 antibody as previously described (McMullin and Hallberg 1988), and specific immune complexes were detected by chemiluminescence using horseradish peroxidase conjugated to Protein A (Amersham ECL kit).

Fluorescence microscopy. Logarithmically-growing cells were prepared for immunofluorescence microscopy essentially as described by Pringle et al. (1991). Nuclei were stained with 4,6-diamidino-2- 


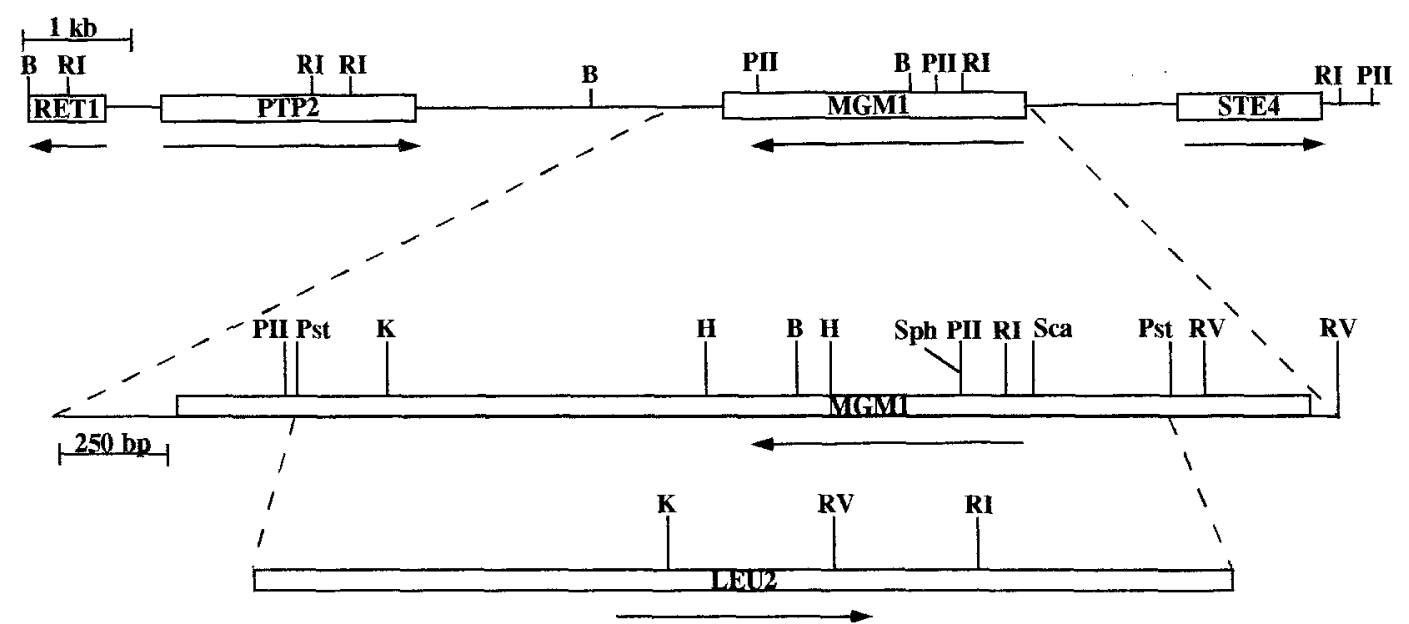

Fig. 1. Physical map of Saccharomyces cerevisiae chromosome XV in the region of $M G M 1$. The top map is the $14-\mathrm{kb}$ region of the right arm of chromosome XV that surrounds $M G M 1$. The genes include a subunit of RNA polymerase III ( $R E T 1$ ), a protein tyrosine phosphatase (PTP2), a $\beta$ subunit of a heterotrimeric G-protein (STE4), and $M G M 1$. The restriction enzyme sites are: BamHI $(B), E c o$ RI $(R I), P v u \mathrm{II}(\mathrm{PII}), P s t \mathrm{I}\left(P_{s t}\right), K p n \mathrm{I}(K), H i n d I I I(H), S p h \mathrm{I}(S p h), S c a \mathrm{I}$ $(S c a)$, and EcoRV (RV). The two bottom maps show how a $2.2-\mathrm{kb}$ $L E U 2$ fragment was used to create a gene replacement between the two PstI sites of $M G M 1$ phenylindole (DAPI, Sigma) at a final concentration of $40 \mathrm{ng} / \mathrm{ml}$ for $5 \mathrm{~min}$, followed by three washes with PBS-BSA (phosphatebuffered saline containing $10 \mathrm{mg} / \mathrm{ml}$ bovine serum albumin). Mitochondria were stained with 2-(4-dimethylaminostyryl)-1-methyl pyridinium iodide (DASPMI) by published procedures (McConnel et al. 1990). Immunofluorescent staining of mitochondria was performed using an immunopurified, anti-hsp58 polyclonal antibody (1:500 dilution), kindly provided by Dr. Richard Hallberg (State University of New York-Syracuse). Rhodamine-conjugated goat anti-rabbit IgG (Cappel Laboratories), diluted 1:1000 in PBS-BSA was used to visualize specific immune complexes. Cells were examined by phase-contrast and fluorescence microscopy using either a Zeiss Axioscope, or a BioRad MRC-600 confocal microscope utilizing a $568 \mathrm{~nm}$ laser source to detect rhodamine conjugates.

\section{Results}

\section{Isolation and sequence analysis of yeast MGM1 gene}

The $M G M 1$ gene was isolated as part of a genomic fragment of chromosome XV that carries RET1, PTP2, $M G M 1$, and STE4 within a contiguous $14-\mathrm{kb}$ region (Whiteway et al. 1990; James et al. 1991; Guan et al. 1992; Jones and Fangman 1992). A map of this region is shown in Fig. 1. The DNA sequence of $3.015 \mathrm{~kb}$ surrounding the $M G M 1$ gene was determined (Fig. 2). A single large open reading frame was found beginning with ATG at nucleotide 1 and ending with a TGA stop codon at nucleotide 2706 . This open reading frame potentially encodes a protein of 902 amino acids with a predicted molecular mass of $101.5 \mathrm{kDa}$. We have tentatively assigned this ATG as encoding the initiator Met because no other in-frame ATG occurs upstream before encountering a stop. It is unlikely that an intron interrupts this region because there are no consensus splice sites within it and an MGM1-specific RNA of $3.0 \mathrm{~kb}$ is detected by a Northern blot, which is in agreement with the predicted size of the $M G M 1$ transcript (Fig. 3). A second in-frame ATG codon occurs at the 22 nd codon of the open reading frame and we cannot be certain at this time which of these methionines initiates translation. If the first ATG is used, the protein would be 902 amino acids whereas if the second is used, the protein would be 880 residues long. In either case, the protein product predicted by our sequence is larger than the 843 amino acids reported earlier (Jones and Fangman 1992). After consulting with the authors, we have confirmed that the previous report contained a sequence error and the correct sequence is as shown in Fig. 2.

\section{The MGM1 gene encodes a protein with homology to the GTP binding domain of dynamin}

As pointed out by Jones and Fangman (1992), Mgm1p shares significant sequence homology with rat dynamin (D100) (Obar et al. 1990), mouse interferon-inducible gene Mx1 (Nakayama et al. 1991), and the yeast dynamin-like protein, $\mathrm{Vps} 1 \mathrm{p}(\mathrm{Spo} 15 \mathrm{p})$ (Rothman et al. 1990; Yeh et al. 1991). The region of Mgm1p most similar to other dynamins includes a near perfect match with the consensus sequence required for GTP binding. The binding site consists of three elements; Element I (GX GKS) participates in binding phosphate, Element II (DXXG) coordinates $\mathrm{Mg}^{2+}$, and Element III (TKXD) binds the nucleotide base (Dever et al. 1987). These elements are found in Mgm1p beginning with residues 238, 338 and 406 , respectively (Fig. 2). It is interesting to note that the spacing of the conserved elements varies between members of the family. There is a 50 amino-acid insertion between Element I and II in Vps1p that is missing in Mgm1p and D100. The percentage of identical residues is $48 \%$ between Mgm1p (residues 220-440) and either D100 (residues 23-239) or Vps1p (residues 19-282). The homology extends beyond the GTP binding domain at a lower, but significant, level. Excluding the non-homologous amino-terminus of Mgm1 $\mathrm{p}$, the sequence identity is $26 \%$ and $25 \%$ with D100 or Vps1p, respectively (Jones and Fangman 1992). The optimal alignment oc- 
gatcaatatgcaagttgttaatat tccttgtaccgttaaaaacaaaatagtagcgttctattgaaaggcaagacataatcagctaggaa

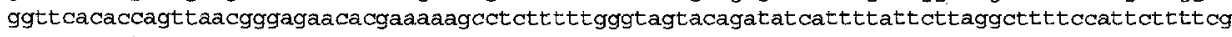
tact tataatatagcct cgcatat caccat aatactctgaaagcATGAGTAATTCIACITCATTAAGGGCCATCCCAAGAGTGGCGAAC $\begin{array}{lllllllllllllllllllllll}M & S & N & S & T & S & L & R & A & I & P & R & V & A & N\end{array}$ TATAACACATTAGMAGGATGAATGCGAGCCCAGTACGGCITTPAAT TTGAGAAGACAGCTTGCTACGCATCCGGCGATACTATATAGC $\begin{array}{llllllllllllllllllllllllllllllll}Y & N & T & L & V & R & M & N & A & S & P & V & R & L & L & I & L & R & R & Q & L & A & T & H & P & A & I & I & Y & S\end{array}$ TCTCCTTACATCAAAAGTCCGITGGTGCACTIACACAGCCGCATGAGCAATGTTCACAGATCAGCACATGCCAATGCITTGAGTITITTI

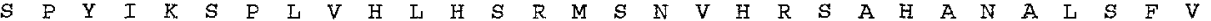
ATCACCAGGAGATCGATATCACATTTTCCTAAAATTATATCTAAAATTATTAGGTPACCCATATATGTGGGTGGCGGGATGGCTGCTGCA $\begin{array}{llllllllllllllllllllllllllllllll}I & T & R & R & S & I & S & H & F^{\prime} & P & K & I & I & S & K & I & I & R & L & P & I & Y & V & G & G & G & M & A & A & A\end{array}$ GGGAGTTATATAGCTIATAAGATGGAAGAAGCTAGCTCITTTACTAAGGACAAACTAGATCGAATCAAGGATPTAGGTGAATCGATGAAG $\begin{array}{lllllllllllllllllllllllllllllll}G & S & Y & I & A & Y & K & M & E & E & A & S & S & F & T & K & D & K & L & D & R & I & K & D & L & G & E & S & M & K\end{array}$ GAAAAGTITAATAAAATGTITTCGGTGATAAGTCACAAGACGGTGGTCATGGAAACGATGGAACGTGCCAACTGCTACTCTAATAGCC

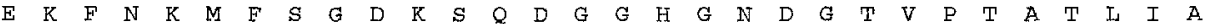
GCIACATCACTCGACGATGACGAAAGIAAGAGGCAAGGAGATCCTAAAGATGATGACGACGAAGATGATGATGACGAGGAIGATGAAAAC

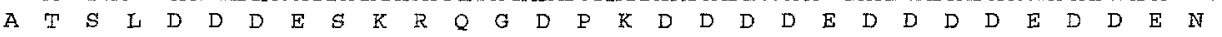
ACCAAAGATTCTGTAGATACAACGCAAGACGAAATGTTAAACTTACAAATGATTGAAAT IAGAACTATTHTGAATAAAGTGGACTCTTCT

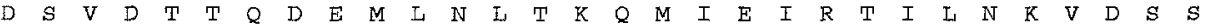
TCTGCACATP TAACTTPACCATCTATTGTCGTGATAGGTTCACAATCGTCTGGTAAATCCTCAGTACTAGAATCCATTGTPGGGAGGGAA

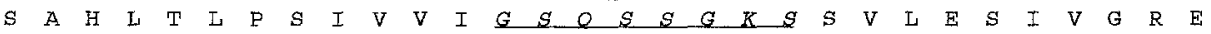
TTCITACCAAAAGGTTCCAACATGGTCACAAGAAGACCCAITGAATTGACTITGGTCAATACACCTAACTCGAATAATGTAACAGCTGAT

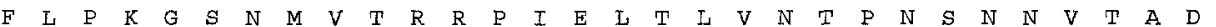
TTTCCAAGCATGCGTCTATACAAIATCAAGGACTITAAAGAGGTGAAAAGAATGCTAATGGAACTGAACAIGGCCGTTCCGACTTCAGAG $\begin{array}{lllllllllllllllllllllllllllllll}F & P & S & M & R & L & Y & N & I & K & D & F & K & E & V & K & R & M & I & M & E & L & N & M & A & V & P & T & S & E\end{array}$ GCTGITTCAGAGGAGCCTAITCAATIGACAATCAAATCGTCCCGCGITCCAGAITTATCAITGGTGGATCIACCIGGCTACATACAAGTG

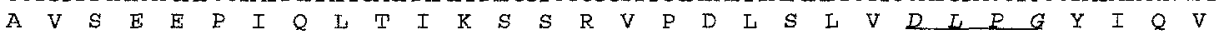
GAAGCGGCAGACCAGCCAAT'AGAACTGAAAACAAAAATCCGTGACCTCTGCGAAAAATATTTGACTGCACCTAATAT"TATTTWAGCAATT

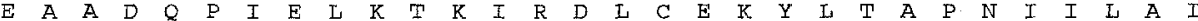
TCRGCTGCGGATGTIGACCTGGCTAATAGTTCIGCITTGAAAGCTPCTAAAGCGGCAGATCCTAAAGGCITGAGAACGATAGGTGTCATT $\begin{array}{lllllllllllllllllllllllllllllll}S & A & A & D & V & D & L & A & N & S & S & A & L & K & A & S & K & A & A & D & P & K & G & L & R & T & I & G & V & I\end{array}$ ACCAAACTGGATT'TGGGGATCCTGAAAAAGCAAGAAGCATCITGAATAATAAAAAATATCCTCTCAGTATGGGCIATGTAGGAGTGATI $\begin{array}{llllllllllllllllllllllllllllllll}I & K & L & D & \mathrm{~L} & \mathrm{~V} & \mathrm{D} & \mathrm{P} & \mathrm{E} & \mathrm{K} & \mathrm{A} & \mathrm{R} & \mathrm{S} & \mathrm{I} & \mathrm{L} & \mathrm{N} & \mathrm{N} & \mathrm{K} & \mathrm{K} & \mathrm{Y} & \mathrm{P} & \mathrm{L} & \mathrm{S} & \mathrm{M} & \mathrm{G} & \mathrm{Y} & \mathrm{V} & \mathrm{G} & V & I\end{array}$ 作 $\begin{array}{llllllllllllllllllllllllllll}Q & S & S & G & E & E & N & T & N & G & i & K & Q & I & V & S & H & Q & F & E & K & A & Y & F & K & E & N\end{array}$ AAGTATITACCAATTGCCAAGTTCCACTAAGAAATIGAGAGAAAAGTTGATCAAAATCITGGAGAT'TCCATGTCAAATGCGCTG 1575 $\begin{array}{llllllllllllllllllllllllllllllll}K & K & Y & F & T & N & C & Q & V & S & T & K & K & L & R & E & K & L & I & K & I & L & E & I & S & M & S & N & A & L & & (525)\end{array}$ GAGCCGACTTCAACACIPATPCAACAAGAGITGGAIGATACITCTIATITGITIAAAGTAGAGITPAACGACAGGCATCTAACTCCTAAA 1665 $\begin{array}{llllllllllllllllllllllllllllllll}\mathrm{E} & \mathrm{P} & \mathrm{T} & S & \mathrm{~T} & \mathrm{I} & \mathrm{I} & \mathrm{Q} & Q & \mathrm{E} & \mathrm{I} & \mathrm{D} & \mathrm{D} & \mathrm{T} & \mathrm{S} & \mathrm{Y} & \mathrm{L} & \mathrm{F} & \mathrm{K} & \mathrm{V} & \mathrm{E} & \mathrm{F} & \mathrm{N} & \mathrm{D} & \mathrm{R} & \mathrm{H} & \mathrm{L} & \mathrm{T} & \mathrm{P} & \mathrm{K} & \text { (555) }\end{array}$ TCAIACCITTTGAATAATATAGATGTACIAAAGTTGGMIITAAAGAGTTICAGGAAAAAITTCATAGAAATGAACTGAAATCAATCTIG 1755 $\begin{array}{llllllllllllllllllllllllllllllll}S & Y & L & I & N & N & I & D & V & L & K & L & G & I & K & E & F & Q & E & K & F & H & R & N & E & L & K & S & I & I & & (585)\end{array}$ AGAGCAGAATTGGATCAGAAAGTTTAGATGTITTGGCTACACGGDATTGGAAGGAIGATAATCTTCAGGATCTATCTTCCTCTAAGCTG 1845 $\begin{array}{llllllllllllllllllllllllllllllllll}R & A & E & I & D & Q & K & V & L & D & V & I & A & T & R & Y & W & K & D & D & N & I & Q & D & L & S & S & S & K & I & & (615)\end{array}$ GAAAGTGATACCGATATGCTATATTGGCATAAAAAATTGGAACTCGCATCATCCGGCITAACTAAAATGGGCATTGGTAGATTATCTACA 1935 $\begin{array}{llllllllllllllllllllllllllllllll}E & S & D & T & D & M & L & Y & W & H & K & K & L & E & I & A & S & S & G & L & T & K & M & G & I & G & R & L & S & T & & (645)\end{array}$ ATGCTMACTACCAATGCAATTTIAAAGGAGCTAGATAACATTCTGGAGTCTACTCAACTAAAAAACCATGAACTGATCAAACACCTTGTT 2025

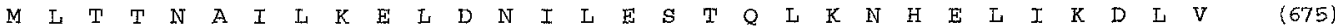
AGCAATACAGCCATTAACGTTCTAAACAGTAAATACTAITCAACGGCTGATCAAGTTGAGAACTGTATCAAACCCTTCAAGTATGAAATI 21.15 $\begin{array}{llllllllllllllllllllllllllllllll}S & N & T & A & I & N & V & I & N & S & K & Y & Y & S & T & A & D & Q & V & E & N & C & I & K & P & F & K & Y & E & I & & (705)\end{array}$ GATTTGGAAGAAAGAGACTGGAGCCTTGCTCGTCAACATTCCATTAATTTAATAAAGGAAGAATTACGTCAATGTAACTCAAGGTACCAG 2205 $\begin{array}{llllllllllllllllllllllllllllllll}D & I & E & E & R & D & W & S & L & A & R & Q & H & S & I & N & L & I & K & E & E & I & R & Q & C & N & S & R & Y & Q & & (735)\end{array}$ GCAATCAAAAATGCCGTTGGCAGTAAGAAATTAGCAAATGTAATGGGCTATITAGAAAATGAATCAAAITIGCAAAAAGAAACACTOGGC 2295 $\begin{array}{llllllllllllllllllllllllllllllll}A & I & K & N & A & V & G & S & K & K & L & A & N & V & M & G & Y & L & E & N & E & S & N & L & Q & K & E & T & L & G & (765)\end{array}$ ATGTCCAAATIACTAITGGAAAGAGGCTCTGAAGCTAIPTWCCTGGALAAAAGGTGCAAAGTGTTATCGTTCAGAITAAAAATGTTGAAA 2385 $\begin{array}{llllllllllllllllllllllllllllllll}M & S & K & \text { I } & L & L & E & R & G & S & E & A & I & F & I & D & K & R & C & K & V & L & S & F & R & L & K & M & L & K & & (795)\end{array}$ AACAAATGPCATPCGACAATTGAGAAGATCGTTGTCCAGAGGTATTPCTATCTGCAGTTAGTGATAAATTGACCTCCACAGCTGTITTA 2475 $\begin{array}{lllllllllllllllllllllllllllllllll}N & K & C & H & S & T & I & E & K & D & R & C & P & E & V & F & L & S & A & V & S & D & K & L & T & S & T & A & V & L & & (825)\end{array}$

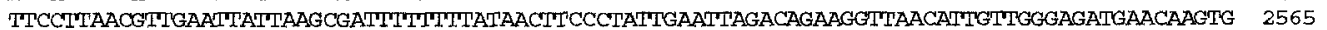

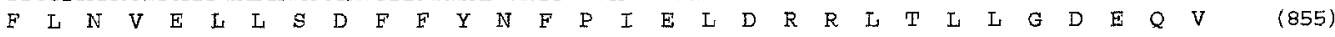
GAAATGITTGCTAAGGAAGATCCGAAGATTTCAAGGCATATTGAATTGCAAAAGAGAAAGGAGCTGTTAGAATTAGCCTMGGAGAAAATA 2655

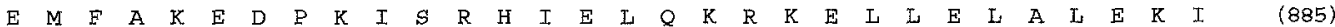
GATPCAATCTTGGTPTPCAAGAAAAGCTACAAGGGCGPCTCCAAAAATTTATGAtgtaaaataaatagtgtcattagagaatttgtaat 2745 $\begin{array}{lllllllllllllllllllllllll}D & S & I & L & V & F & K & K & S & Y & K & G & V & S & K & N & L & \text { * }\end{array}$ agcatttttaccgttatataattactagggtgtattttctgaaaaaataaaggcataccctccatctaagctcttcttatagtatac 2835 sagtctcttatatcgcacgatgtaacatctacatacttcctttatcgtcttgaagagtaaacaaaagatatatatatattattttctatt 2925 atttggaagagaatggtattcctacagtatgcagagacacatctggttcatacttcctttatcgtcttgaagagtaaacaaaagatata 3015

Fig. 2. DNA and deduced amino-acid sequence of $M G M 1$. The DNA sequence of 3015 bases encompassing $M G M 1$ was determined from both strands of overlapping fragments subcloned into either M13mp18 or M13mp19 phage (see Materials and methods). The proposed $M G M 1$ protein-coding region begins at nucleotide 1 and terminates at nucleotide 2706 . Nucleotide and amino-acid numbers

curs when the carboxy-terminal three quarters of Mgm1p is lined up with the amino-terminal three quarters of D100 and Vps1p.

The amino-terminus of Mgm1 $\mathrm{p}$ is very basic; there are 15 lysines and arginines, but no acidic residues, in the first 113 residues. In addition, this region is rich in hydroxylated amino acids ( 17 serine and threonine). These are char- (in parenthesis) are given on the right side of the sequence. Three conserved amino-acid sequence elements characteristic of proteins binding GTP are indicated by underlined italics in the sequence. These sequence data are available from EMBL/GenBank/DDBJ under accession number L07419

acteristics of proteins that are localized to the mitochondria (von Heijne 1986; Glick and Schatz 1991). Although we currently do not know whether Mgm1p localizes to the mitochondria, the mitochondrial defect of the $m g m 1$ mutant (see below) makes this an attractive possibility to consider. The basic amino-terminal domain of $\mathrm{Mgm} 1 \mathrm{p}$ is connected to the rest of the protein by a stretch of 13 


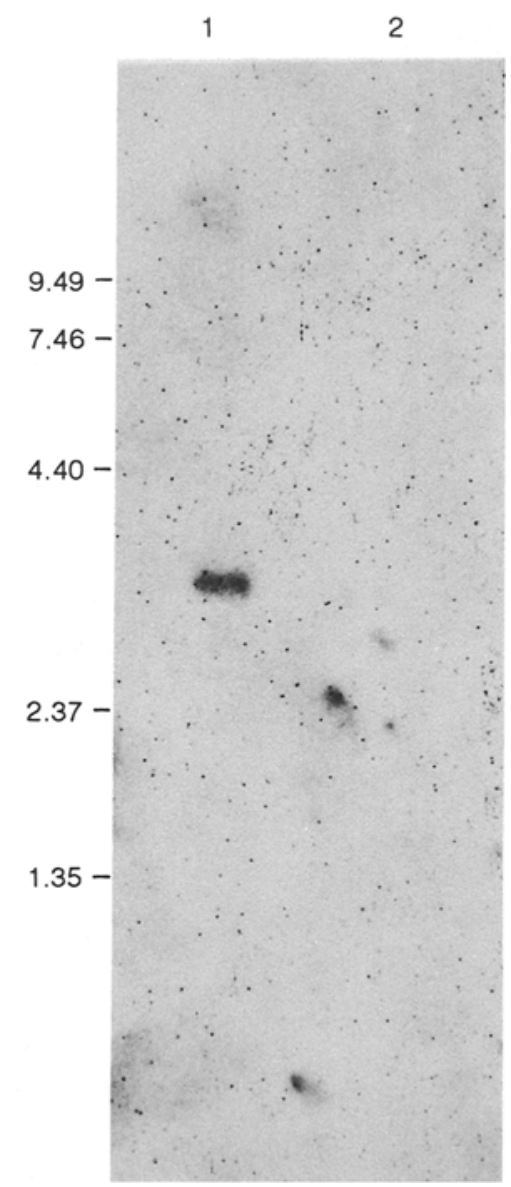

Fig. 3. Northern hybridization of poly $\mathrm{A}^{+}$RNA isolated from RJY310. RNA isolated from RJY310 was blotted to nitrocellulose and probed with a $2.5-\mathrm{kb}^{32} \mathrm{P}$-labeled probe generated by labeling a PCR fragment which covers nearly the entire $M G M 1$ gene. Ethidium bromide-stained RNA standards (BRL) were loaded to estimate transcript sizes. Lane 1 is $5 \mu \mathrm{g}$ of poly $\mathrm{A}^{+}$yeast RNA and lane 2 is $5 \mu \mathrm{g}$ poly $\mathrm{A}^{-}$RNA (RNA not bound to the Oligo-dT column). The gel was exposed for 3 days with an intensifying screen using Kodak X-Omat AR film

acidic residues. The significance of this cluster is not known.

\section{The mitochondrial genome is absent in strains lacking Mgm1p}

The loss of $M G M 1$ results in loss of the mitochondrial genome (Jones and Fangman 1992). To analyze further the consequence of mutating $M G M 1$, we constructed an MGM1 gene disruption by inserting $L E U 2$ into the protein-coding region. The resulting heterozygous diploid was sporulated and the tetrads were dissected. Figure 4A shows a Southern blot confirming that the gene was disrupted and the expected 2:2 segregation of the two alleles occurs. Colonies arising from the tetrad dissection segregate into large and small when plated onto YEPD (Fig. 4B). In each case, the small colonies corresponded to strains prototrophic for leucine indicating that they arose from the MGM1 null strains. The slowgrowth phenotype was shown to be the result of a mito-

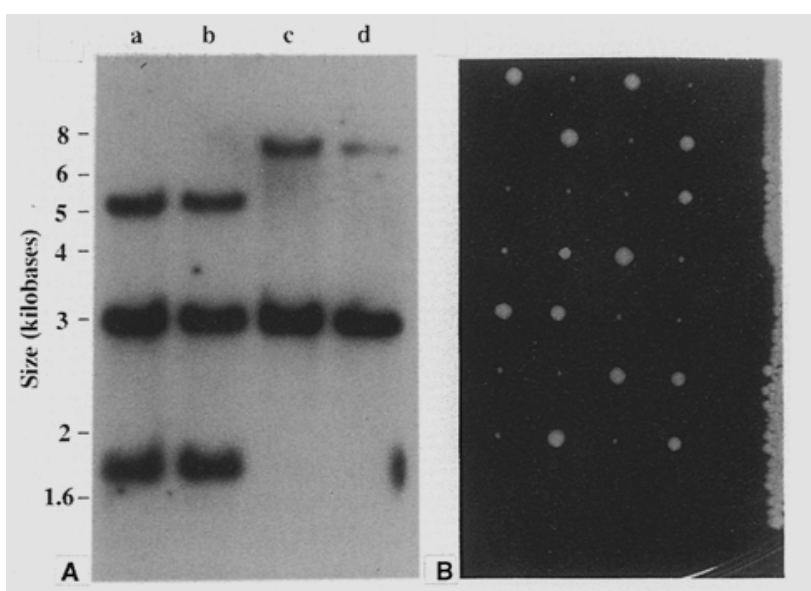

Fig. 4A, B. Characterization of an $M G M 1$ gene replacement. Strain RJY389 was transformed with pMGM1::LEU2 to create RJY463, a heterozygous $M G M 1 / m g m 1:: L E U 2$ diploid. RJY463 was sporulated and tetrads were dissected. Panel $\mathbf{A}$ is a Southern blot of DNA isolated from a typical tetrad. The DNA was digested with $P v u I$ I, resolved on a $1.0 \%$ agarose gel, blotted to nitrocellulose and hybridized with a ${ }^{32} \mathrm{P}$-labeled pMGM1 probe. Panel B shows the colonies from the tetrad dissection revealing 2:2 segregation of the petite-growth phenotype. The cells are plated on YEPD medium

chondrial defect by plating cells on medium containing a non-fermentable carbon source (YEP- $\%$ glycerol $/ 2 \%$ ethanol); (Jones and Fangman 1992). To further examine the mitochondrial defect associated with deleting $M G M 1$, we crossed RJY386 to a $\varrho^{\circ}$ strain, KL14-4B. If the mitochondrial DNA is unaffected by the loss of $M G M 1$, then the diploid created between RJY386 and KL14-4B should be respiratory competent. However, we found that diploids arising from this cross remained respiratory deficient (data not shown). Therefore, loss of Mgm1 $p$ results in a complete loss of the mitochondrial genome $\left(\varrho^{\circ}\right)$.

\section{Abnormal mitochondrial-morphology results from deleting MGM1}

Nuclear and mitochondrial DNA was examined by staining cells with DAPI (Fig. 5). Mitochondrial DNA is readily observed in wild-type yeast (RJY389) stained with DAPI, appearing as punctate staining, localized throughout the cytoplasm of the cell (Fig. 5, panel b). As expected, based on the genetic assays described above, mitochondrial DNA staining was not observed in RJY390, in which MGM1 has been deleted (Fig. 5, panel d). Therefore, two lines of evidence, one genetic and the other involving direct microscopic visualization with DAPI, reveal that deletion of $M G M 1$ results in the complete loss of the mitochondrial genome.

We attempted to visualize mitochondria with DASPMI, a dye that fluoresces when it partitions into functional mitochondria (Bereiter-Hahn 1976). Wild-type cells (RJY390) stained with DASPMI in a pattern that was distributed similarly to the DAPI staining described 

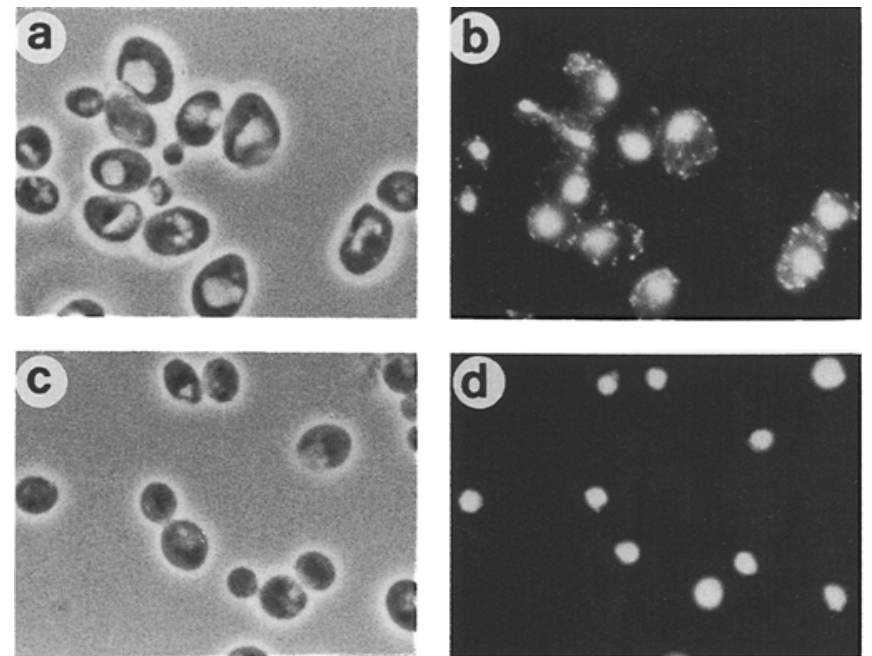

Fig. 5a-d. Light micrographs of DNA staining of wild-type and mgm1 mutant cells. A wild-type MGM1 diploid, RJY389 (panels a, b) and an $m g m 1$ mutant diploid, RJY390 (panels c, d), were visualized by phase-contrast microscopy (panels $\mathbf{a}, \mathbf{c}$ ) or following staining with DAPI (panels b, d)

above. However, staining was not observed in RJY390, which lacks Mgm1p, indicating that the mitochondria in these strains are defective (data not shown). Therefore, an immunofluorescence method that does not require that the mitochondria are functional was used to visualize mitochondria. Cells were reacted with antibody to hsp58p, a yeast heat-shock protein that is localized to the mitochondrial matrix (McMullin and Hallberg 1988). The mitochondria of wild-type cells (RJY389) appear as bright spots, dispersed in tracts throughout the cytoplasm of both mother and bud (Fig. 6, panel a). The same general pattern is observed in the $\varrho^{\circ}$ diploid strain, RJY545 (Fig. 6, panel b). However, strains lacking Mgm1p exhibited a dramatically different pattern. The fluorescence signal was concentrated in dense clumps, rather than in the dispersed pattern observed in the wildtype (Fig. 6, panel c). The mitochondrial-like structures in the mutant partition to the daughter cells at cell division.

We have not conclusively determined that the hsp58 staining in the mutant corresponds to mitochondria. However, one indication that hsp $58 \mathrm{p}$ localizes to a mitochondrial compartment, even in the mutant, comes from an analysis of the protein by Western blotting (Fig. 7). Import of hsp $58 \mathrm{p}$ into the mitochondrial matrix is associated with proteolytic processing of an amino-terminal localization signal (McMullin and Hallberg 1988). When a Western blot of protein from wild-type cells (RJY389) was probed with anti-hsp $58 \mathrm{p}$, a single immuno-reactive band of approximately $58 \mathrm{kDa}$ was observed (Fig. 7), whereas in the mutant two bands were present. The bottom band of the doublet co-migrated with mature hsp $58 \mathrm{p}$, suggesting, but not proving, that hsp58p is processed and transported into mitochondria-like structures. The lower efficiency of import could be the result of fewer mitochondria or else of a protein-import defect in the mutant cells.
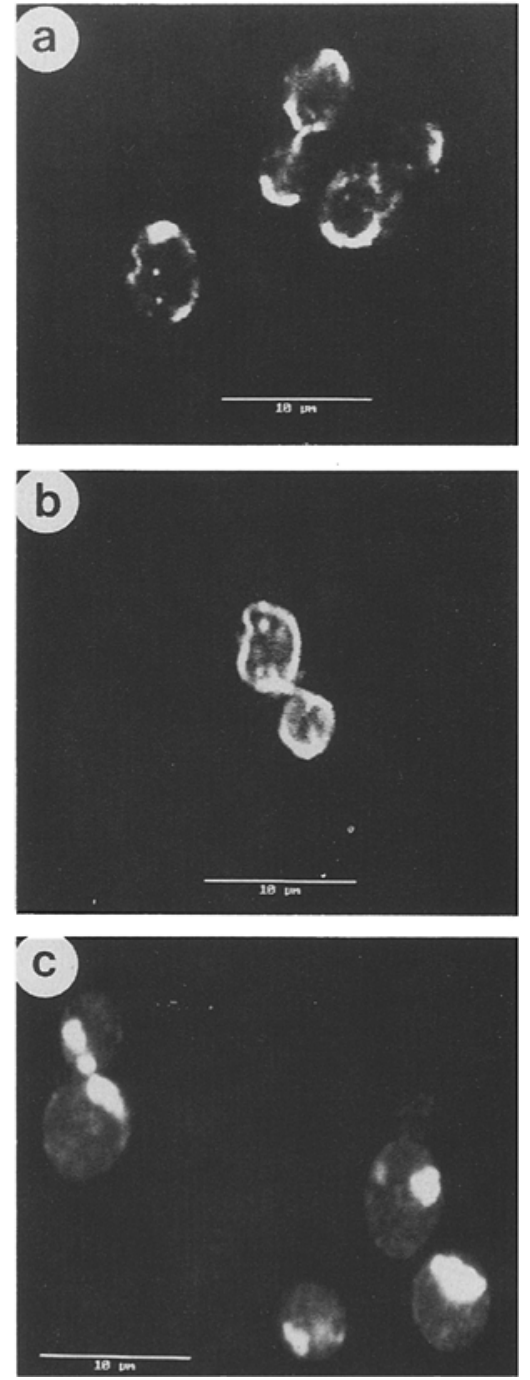

Fig. 6a-c. Immunofluorescence staining of $M G M 1$ wild-type and $m g m 1$ mutant cells with anti-hsp58 antibody. Cells from wild-type, RJY389 (panel a), a $\varrho^{\circ}$ diploid, RJY545 (panel b), or the $m g m 1$ mutant diploid, RJY555 (panel c), were reacted with anti-hsp58 antibody and observed by confocal microscopy. Fluorescent complexes were generated with a secondary antibody, rhodamine-conjugated goat anti-rabbit IgG (Cappel Laboratories)

\section{Discussion}

In this report, we describe our work to further the understanding of a dynamin-related gene $M G M 1$, from $S$. cerevisiae. The $M G M 1$ gene is predicted to encode a $902-$ residue polypeptide with an approximate molecular mass of $101 \mathrm{kDa}$. This size is $7 \mathrm{kDa}$ larger than that predicted by an earlier reported $M G M 1$ sequence (Jones and Fangman 1992). Consistent with the results of Jones and Fangman (1992), creation of a null allele of $M G M 1$ causes a mitochondrial defect that leads to the loss of the mitochondrial genome. We also found that deleting $M G M 1$ resulted in a dramatic structural alteration in the mitochondria and a defect in protein import, as evidenced by the accumulation of a mitochondrial protein precursor. 


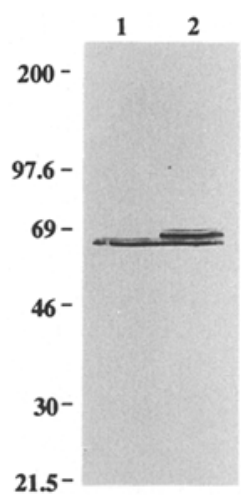

Fig. 7. Immunological detection of hsp 58 protein in extracts of wild-type and $m g m 1$ mutant cells. Total cellular protein was isolated as described in the Materials and methods. Approximately $50 \mu \mathrm{g}$ of protein from RJY389 (lane 1) or RJY390 (lane 2) was resolved by SDS-PAGE on a $12.5 \%$ gel. The gel was electroblotted to nitrocellulose and probed with anti-hsp58 antibody (1:2000 dilution). Following washing, specific complexes were detected with HRP-conjugated Protein A and chemiluminescence. The migration position of a set of prestained molecular weight markers is given in kilodaltons to the left of the gel. Hsp58 runs with an apparent molecular weight larger than the reported size (McMullin and Hallberg 1988)

Although the original dynamin protein, D100, was isolated based on its nucleotide-dependent interaction with microtubules, there is little direct evidence that dynamins function as microtubule-based motors. Analysis of the mutant phenotypes of Drosophila shibire and yeast VPS1 suggests a role for dynamin proteins in intracellular vesicle movement. The finding that another member of the family, $M G M 1$, is involved in mitochondrial activity raises additional questions regarding the function of these proteins. Is there a role for microtubules in mitochondrial function? Electron micrographs showing microtubules in close proximity to mitochondria have been observed suggesting that the cytoskeleton may play a role in some aspect of mitochondrial function (Ball and Singer 1982; Lin et al. 1990). One possibility is that microtubules and $\mathrm{Mgm} 1 \mathrm{p}$ are needed for intracellular movement of mitochondria. Mutations have been described that effect mitochondrial partitioning at cell division (McConnell et al. 1990). However, in yeast, the transport of mitochondria into the bud is independent of microtubules (Huffaker et al. 1988; Jacobs et al. 1988). Our results are not consistent with a role for $\mathrm{Mgm} 1 \mathrm{p}$ in mitochondrial partitioning. If deletion of $M G M 1$ interfered with mitochondrial inheritance, then a heterogeneous population of cells would result. Some cells would have mitochondrial function, while others would be respiratory deficient. However, DAPI staining revealed a uniform lack of mitochondrial DNA staining in a population of $m g m 1$ mutant cells. In addition, the genetic crosses between the mgm 1 deletion strain and the $\varrho^{\circ}$ tester would have resulted in complementation of the respiration defect, and this was not observed. Therefore, the defect is not due to uneven segregation of a population of functional mitochondria, but rather the mitochondria have undergone a uniform loss of mitochondrial DNA as a result of mutating the $M G M 1$ gene.
A direct role for Mgm1p in mitochondrial DNA synthesis predicts that it is localized to the mitochondria. The subcellular localization of Mgm $1 \mathrm{p}$ is not known, but the amino-terminal sequence of the protein has a number of features found in mitochondrial localization signals. Proteins destined for the mitochondria are usually rich in basic and hydroxylated amino acids, lack acidic residues, and may form amphiphilic helixes (von Heijne 1986; Glick and Schatz 1991). Two regions near the amino-terminus of Mgm1p exhibit these features. One region, between residues 8-26, contains three Arg, two hydroxylated amino acids, and no acidic residues. If this region is modeled as an $\alpha$ helix and scored for its amphiphilic character by the method of von Heijne (1986), a $\mu_{\mathrm{H}}$ value of 5.1 is obtained. Values ranging from 5.1 to 12.5 have been reported for proteins in which the mitochondrial localization signals have been mapped. The second region that might constitute a mitochondrial targeting sequence occurs between residues 78 and 95 . Its $\mu_{\mathbf{H}}$ value for amphiphilicity is 13.7 , which is even better than values reported for authentic mitochondrial localization sequences. This region was recognized by Jones and Fangman (1992) to be a potential mitochondrial targeting sequence. However, the corrected sequence predicts that this region is farther from the amino-terminus of the protein than originally thought, whereas mitochondrial localization sequences are generally closer to the aminoterminus (Glick and Schatz 1991). A more favorable positioning of this putative mitochondrial targeting sequence could occur if one of the internal methionines initiated translation. Examples of alternative initiator methionine utilization creating mitochondrial and nonmitochondrial forms of a protein have been reported (Beltzer et al. 1988).

A second way in which Mgm1p could affect the mitochondrial genome may be indirect. For example, Mgm1p may be required for the efficient import of proteins required for replication or partitioning of the mitochondrial genome. A total block in protein import is not expected because mutations in genes that totally abolish mitochondrial import are lethal (Yaffe and Schatz 1984). However, partial inhibition of the import of a critical factor needed for replication or partitioning of the mitochondrial genome could result in the generation of the $\varrho^{\circ}$ phenotype we observe. One indication that mitochondrial protein import is affected is the observed accumulation of hsp 58 precursor in the $m g m 1$ mutant. Transport of hsp 58 is accompanied by cleavage of the amino-terminal leader sequence. We find that this processing is slower in the $m g m 1$ mutant, suggesting that transport is defective in the absence of $M G M 1$. Alternatively, the inefficient protein transport might be explained by fewer mitochondria rather than by a specific defect in the import pathway. We are unable to distinguish between these possibilities at this time.

Yeast mitochondria undergo dramatic changes in size, shape, and DNA content, depending on growth condition (Dujon 1981; Stevens 1981). Rapidly-growing cells that are glucose repressed have few mitochondria and they are dispersed in the peripheral cytoplasm. Under conditions of aerobic growth, the percentage of the cyto- 
plasm occupied by mitochondria increases from $3 \%$ to $12 \%$ and the mitochondrial DNA can increase five-fold (Stevens 1981). Proliferation of mitochondria is accomplished by fusion events that form a reticular network of mitochondria. The signals that trigger this event and the biosynthetic machinery that must be activated to accomplish this elaborate proliferation are not understood. Mgm1p could play a role in this process. Two of the dynamin proteins that have been described play a role in vesicle trafficking. Drosophila shibire affects endosomes while yeast VPS1 protein affects the organization of membranes required for vacuole formation. Although vacuoles appear normal in mgmt mutant cells (R.J.D., unpublished results), the intracellular distribution of mitochondria is altered in the mutant. Instead of the fairly uniform, dispersed appearance of the mitochondria around the periphery of the cell, mitochondria in the mutant appear in condensed structures (Fig. 6). Perhaps $\mathrm{Mgm} 1 \mathrm{p}$ plays a role in the biogenesis or reorganization of mitochondria following a change in carbon source. Genome loss might be a secondary consequence of that defect.

Acknowledgements. We are grateful to Mary Crivellone for supplying the $\varrho^{\circ}$ yeast strains, Richard Hallberg for the anti-hsp58 antibodies, Frank Longo for help with microscopy, and Thomas Moninger of the University of Iowa Central Electron Microscope Research Facility for obtaining the confocal images. We also thank Dan Weeks, Peter Rubenstein and members of the laboratory for helpful comments on the manuscript. We would like to especially acknowledge the enthusiastic support of Dr. Jack Dixon during all phases of this study. This work was supported by grants from the National Institutes of Health (CA50211) and the Pardee Cancer Research Fund to R.J.D., and the University of Michigan Geriatics Center to K.G.

\section{References}

Ball EH, Singer SJ (1982) Proc Natl Acad Sci USA 79:123-126 Beltzer JP, Morris SR, Kohlhaw GB (1988) J Biol Chem 263:368374

Bereiter-Hahn J (1976) Biophys Acta 423:1-14

Bliek AM van der, Meyerowitz EM (1991) Nature 351:411-414

Chen MS, Obar RA, Schroeder CC, Austin TW, Poodry CA, Wadsworth SC, Vallee RB (1991) Nature 351:583-586

Deschenes RJ, Broach JR (1987) Mol Cell Biol 7:2344-2351

Dever TE, Glynias MJ, Merrick WC (1987) Proc Natl Acad Sci USA $84: 1814-1818$

Dujon B (1981) In: Strathern JN, Jones EW, Broach JR (eds) Molecular biology of the yeast Saccharomyces: life cycle and Inheritance. Cold Spring Harbor Laboratory, Cold Spring Harbor, New York, pp 505-635

Glick B, Schatz G (1991) Annu Rev Genet 25:21-44

Guan K, Deschenes RJ, Qiu H, Dixon JE (1991) J Biol Chem 266: $12964-12970$

Guan K, Deschenes RJ, Dixon JE (1992) J Biol Chem 267:1002410030

Heijne G von (1986) EMBO J 5: 1335-1342
Hoffman CS, Winston F (1987) Gene 57:267-272

Huffaker TC, Thomas JH, Botstein D (1988) J Cell Biol 106: 19972010

Ito H, Fukada Y, Murata K, Kimura A (1983) J Bacteriol 153:163168

Jacobs CW, Adams AEM, Pringle JR (1988) J Cell Biol 107: 1409_ 1426

James P, Whelen S, Hall B (1991) J Biol Chem 266:5616-5624

Jones BA, Fangman WL (1992) Genes Dev 6:380-389

Lin A, Krockmalnic G, Penman S (1990) Proc Natl Acad Sci USA $87: 8565-8569$

McConnell SJ, Stewart LC, Talin A, Yaffe MP (1990) J Cell Biol 111:967-976

McMullin TW, Hallberg RL (1988) Mol Cell Biol 8: 371-380

Myers AM, Crivellone MD, Tzagoloff A (1987) J Biol Chem 262:3388-3397

Nakayama M, Nagata K, Kato A, Ishihama A (1991) J Biol Chem 266:21404-21408

Obar RA, Collins CA, Hammerback JA, Shpetner HS, Vallee RB (1990) Nature 347:256-261

Poodry CA, Edgar L (1979) J Cell Biol 81:520-527

Pringle JR, Adams AEM, Drubin DG, Haarer BK (1991) Methods Enzymol 194:565-602

Rothman JH, Stevens TH (1986) Cell 47:1041-1051

Rothman JH, Raymond CK, Gilbert T, O'Hara PJ, Stevens TH (1990) Cell 61:1063-1074

Rothstein RJ (1983) Methods Enzymol 101:202-211

Sambrook J, Fritsch EF, Maniatis T (1989) In: Molecular cloning: a laboratory manual. Cold Spring Harbor Laboratory, Cold Spring Harbor, New York

Scaife R, Margolis RL (1990) J Cell Biol 111:3023-3033

Schmitt ME, Brown TA, Trumpower BL (1990) Nucleic Acids Res 18: $3091-3092$

Schnapp BJ, Reese TS (1989) Proc Natl Acad Sci USA 86:15481552

Sherman F, Fink GR, Hicks JB (1986) In: Laboratory course manual: methods in yeast genetics. Cold Spring Harbor Laboratory, Cold Spring Harbor, New York, pp 117-159

Shpetner HS, Vallee RB (1989) Cell 59:421-432

Shpetner HS, Vallee RB (1992) Nature 355:733-735

Southern E (1975) J Mol Biol 98:503-517

Stevens B (1981) In: Strathern JN, Jones EW, Broach JR (eds) Molecular biology of the yeast Saccharomyces: life cycle and Inheritance. Cold Spring Harbor Laboratory, Cold Spring Harbor, New York, pp 471-504

Stimmel JB, Deschenes RJ, Volker C, Stock J, Clarke S (1990) Biochemistry 29:9651-9659

Tzagoloff A, Dieckmann CL (1990) Microbiol Rev 54:211-225

Vale RD, Reese TS, Sheetz MP (1985a) Cell 42: 39-50

Vale RD, Schnapp BJ, Mitchison T, Steuer E, Reese TS, Sheetz MP (1985b) Cell 43:623-632

Vallee RB, Shpetner HS, Paschal BP (1989) Trends Neurosci 12: 66 70

Whiteway M, Hougan L, Thomas DY (1990) Mol Cell Biol 10: 217 222

Yaffe MP, Schatz G (1984) Proc Natl Acad Sci USA 81: $4819 \cdots 4823$

Yeh E, Carbon J, Bloom K (1986) Mol Cell Biol 6:158-167

Yeh E, Driscoll R, Coltera M, Olins A, Bloom K (1991) Nature 349:713-715

Communicated by R. J. Rothstein 